\title{
Talking cars, doubtful users - a population study in virtual reality
}

\author{
Farbod N. Nezami ${ }^{\mathrm{a}, 1,2, \bigotimes}$, Maximilian A. Wächter ${ }^{\mathrm{a}, 1}$, Ashima Keshava ${ }^{\mathrm{a}, 3}$, Artur Czeszumski $^{\mathrm{a}, \mathrm{c}, 3}$, Hristofor Lukanov $^{\mathrm{a}, 3}$, Marc Vidal \\ De Palol ${ }^{\mathrm{a}, 3}$, Gordon Pipa ${ }^{\mathrm{a}, 4}$, and Peter König ${ }^{\mathrm{a}, \mathrm{b}, 4}$ \\ ${ }^{\mathrm{a}}$ Institute of Cognitive Science, University of Osnabrück, Osnabrück, Germany \\ ${ }^{b}$ Department of Neurophysiology and Pathophysiology, Center of Experimental Medicine, University Medical Center Hamburg-Eppendorf, Hamburg, Germany \\ ${ }^{c}$ Department of Clinical Psychology, Vrije Universiteit Amsterdam, Amsterdam, The Netherlands
}

\begin{abstract}
Autonomous vehicles as cognitive agents will be an important use case of artificial intelligence in modern societies. Investigating how to increase acceptance and trust, we created a selfexplaining car, informing passengers before actions in virtual reality. This study investigates the attitude towards self-driving cars with data from 7850 participants. We show how gender and age affect the attitude towards autonomous vehicles, resulting in female participants being generally less trusting of overall conditions than male participants and a general decrease of acceptance with increasing age. Surprisingly, a self-explaining car providing the passenger with crucial traffic information, although it has a positive impact on trust but influences the intention of using such a car negatively. Therefore, we argue for a highly individualizable in-car communication that meets the adversarial needs of different demographic groups to enable human-machine interactions that foster safe traffic behavior and increase trust and the willingness to use such technology.
\end{abstract}

Technology acceptance | Autonomous vehicles | Demographic differences | Virtual Reality

Correspondence: fnosratnezam@uni-osnabrueck.de

\section{Introduction}

Autonomous driving vehicles (ADVs) seem to be the primary goal of nearly all car manufacturers since more highly automated driving systems are implemented into newly developed cars (1). Technical developments in the field are fast and continuously improving. There is little doubt that selfdriving cars will have a significant impact on our society, such as drastically decreasing greenhouse gas emissions and reducing the number of traffic-related injuries and fatalities, as well as the shape of the current infrastructure $(2,3)$. However, for these desirable aspects to be realized, it is necessary to use these vehicles in public. This means public acceptance is the critical factor for autonomous vehicles.

According to the current state of surveys, there is surprisingly limited willingness among potential customers to use fully autonomous vehicles (3-7). Various national and global surveys have shown that most of today's car users and potential buyers are unwilling to use a self-driving car or are unwilling to use it to its full extent $(8,9)$. In return, this means that there is currently little market demand for fully autonomous vehicles, which obstructs the realization of the promises this technology holds $(10,11)$. Trust and acceptance of potential customers will shape the extent to which autonomous cars will be implemented into individual traffic $(12,13)$.
Primary reasons for the lack of acceptance are the fear of malfunctions of the systems and fear of giving complete control to the car (14). Further reluctance in adaptation can stem from computer anxiety and low technology self-efficacy (15), meaning how they perceive their capability in operating a high-tech-based system. In other words, drivers do not trust self-driving cars to be safe enough (16). The fundamental issue here might be rooted in the lack of transparency in the self-driving cars' AI systems, namely it is not clear what is and on what basis is their decision in any given situation. Not knowing what the artificial agent perceives and the ambiguity of its course of action directly influences the concern of safety $(17,18)$. Therefore, the communication and transparency between humans and machines may be the key to understanding trust and, consequently, acceptance of self-driving cars (19).

Still, it is not clear yet which factors precisely modulate the acceptance of self-driving vehicles. First, however, the technology must be well accepted to play out possible improvements in traffic. Previous research could already show that too much information about the driving state of a car is distracting $(8-10,12,13,20)$. To gain insight into which factors modulate the acceptance towards ADVs, we developed an interactive large-scale virtual reality (VR) experiment (21), where participants experienced an ADV giving feed-forward and feedback about its current driving status.

Here we gathered questionnaire data from 7850 visitors of a German Ministry of Education and Research traveling exhibition. It is tested whether verbal feed-forward and feedback increase trust and acceptance of autonomous cars. Participants experienced a short car drive in a virtual reality environment in one of three randomly chosen driving conditions: A traditional taxi with a human avatar driving, a self-driving car with the radio playing, and a self-explaining car informing the participant verbally on its actions. During the drive, traffic-related scenarios of potentially unsafe outcomes occurred. The main interest lies in a fine AI condition, where the autonomous car explains its decisions before its actions. We expect to find significant differences in the participants' attitudes between conditions and significant differences between different age groups. To assess the acceptance, we used a shortened version of the Technology Acceptance Model questionnaire (TAM) (22). We investigated trust and acceptance with this modified TAM to obtain insight into factors that modulate trust and acceptance in self-driving vehicles. 


\section{Results}

For six months, questionnaire data from 7850 participants of all ages have been gathered. The data is used to answer questions on the influence of gender and age and the influence of the experimental driving conditions on general acceptance. Each participant observed a 90 seconds drive through a virtual environment (21) in one of the driving conditions as described below:

- A self-driving car with voice assistance informing the participant about the possible dangers ahead and the car's decision. This condition hereon will be called Anthropomorphic voice assistant system (AVAS condition)

- A self-driving car where a podcast plays in the car during the drive. The car behaves precisely the same as in AVAS condition but does not give any information on its decisions to the participant (radio talk condition).

- A ride in a traditional taxi with a female taxi driver, where the participant is placed in the passenger seat and the taxi driver comments on the ride (taxi condition).

Aside from the type of car, all other aspects of the experiment, including the car driving path, dangerous scenarios, and city traffic, are precisely identical. The questionnaire data consist of 4464 participants identified as male and 3386 as female, 400 as intersex, and 349 refusing to identify their gender. Due to the sparse number, people identifying as transgender or refusing to answer are not considered in the analysis (Figure 1 a). Of the participants, 2812 are under the age of 20 years, 1513 between 20 to 40,1883 between 40 to 60,582 between 60 to 80 , and 86 participants above 80 years (Figure $1 \mathrm{~b}$ ). By using optimal binning (23), we ensure homogeneous probability density under each age group. 2691 data sets are recorded in the AVAS condition, 2636 in Taxi Driver condition, and 2509 in Radio Talk (Figure 1 c). A large number of participants in each gender, age bin, and conditions allows the use of regression-like inferential tests (i. e. MANOVA) due to their robustness against non-normalities in large data-sets (24).

This study investigates how and to what extent factors gender, age, and experimental condition affect the intention of use, perceived usefulness, perceived ease of use, and trust in the TAM. During data preparation, it became evident that the questionnaire items show a positive correlation of $r>$ 0.4 . Therefore, these items have to be analyzed together as multivariate dependent variables. MANOVA tests the optimal linear combination of dependent variables to find significant effects. To check validity of the assumptions (nonsignificant p-values), a Levene's test is performed with resulting F-values of: 1.369 for perceived Usefulness ( $\mathrm{p}$-value $=0.089), 2.333$ for Ease of use ( $\mathrm{p}$-value $<0.001), 1.459$ for Intention of use ( $\mathrm{p}$-value $=0.053)$ and 1.443 for Trust $(\mathrm{p}$ value $=0.058$ ). Considering the large sample size, known to reduce p-values in Levene's test, a further check of the covariance matrices for the dependent variables of the TAM

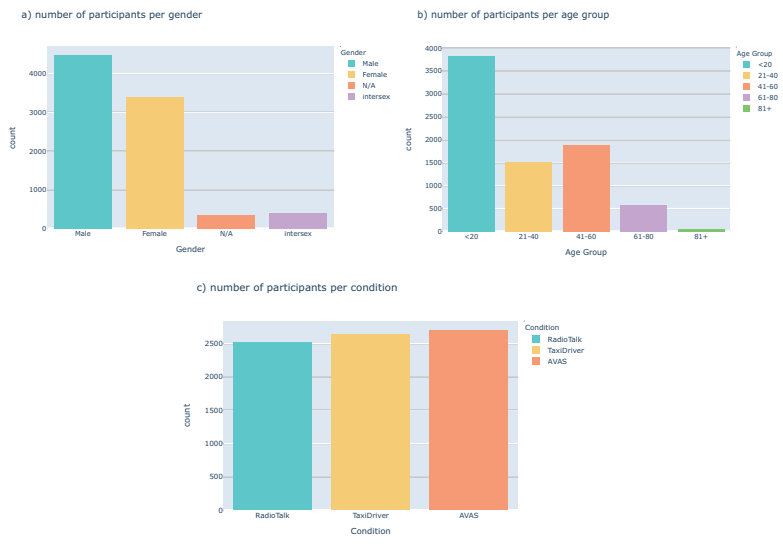

Fig. 1. Histogram of number of participants based on a) gender, b) age group and c) observed driving condition after clean up ( $\mathrm{N}=7850)$.

concerning the main factors of gender, age group, and condition has been done. Together, Levene's test and the covariance matrices provide essential evidence for the validity of the assumptions of MANOVA. Out of the four different null hypothesis tests of the multivariate analysis, Pillai's Trace is chosen due to its known robustness toward non-normalities in the data (25). Therefore, the multivariate analysis of variance is the prime analysis method $(26,27)$.

To gain deeper insights into how gender, age, and condition affect the TAM factors independently, a linear discriminant analysis (LDA) is used to extract each independent variable's weighted influence. Linear discriminant analysis tries to find a set of coefficients that will maximize the separability levels within the given independent variable (i.e., male/female for gender). These coefficients can be used to interpret the influence of each independent variable on each of the modulator factors of TAM (i.e., the influence of gender on perceived usefulness, perceived ease of use, etc.). Therefore LDA allows interpretation of the influence of gender, age group, and condition on the intention of use, perceived usefulness, perceived ease of use, and trust separately.

The effect of gender. To find out the influence of gender on acceptance, the result of the MANOVA with the follow-up LDA specifically for gender is calculated. With a resulting Pillai's Trace $=0.00293 \mathrm{~F}(4,7835)=4.761$, $\mathrm{p}$-value $<0.001$ in the MANOVA, it is evident that there is a significant effect of gender on overall acceptance. The follow-up LDA shows that females have a lower score based on the observed discriminant coefficients. The resulting coefficients are -0.33 for the intention of use, -0.06 for perceived usefulness, -0.60 for perceived ease of use, and -0.18 for trust (Figure 2 a), all with a medium effect size (Cohen's D = 0.45). Additionally, the LDA shows that perceived usefulness and trust are less affected by gender than the intention of use and the perceived ease of use (Figure 2 a). This means that females and males have an almost equivalent attitude towards the perceived usefulness but differ in the perception of ease of use and, consequently, the intention of using self-driving cars. Thus, we interpret that females anticipate difficulties in handling and therefore score also lower in the intention to use this technol- 
ogy.

The effect of age group. Similar to the effects of gender, the result of the MANOVA is paired with a follow-up LDA specifically for the age group to find the influence of the age group on all TAM items. The resulting Pillai's Trace $=0.04561 \mathrm{~F}(16,313)=24.107 \mathrm{p}$-value $<0.001$ indicates the significant effect of age group on the overall acceptance. LDA results in discriminant coefficients of -0.27 for the intention of use, -0.19 for perceived usefulness, -0.46 for perceived ease of use, and -0.30 for trust. These results show that age, in general, has a negative effect on all TAM items. The age group under 20 years of age show high scores, with medium effect sizes compared to the age group between 2040 (Cohen's $\mathrm{D}=0.50$ ) and 60-80 (Cohen's $\mathrm{D}=0.43$ ) (for the full list of the effect sizes, see SI Table1). Like in the previous analysis of gender, perceived ease of use is affected the most (Figure $2 \mathrm{~b}$ ). Together with the decreased intention of use, it can be stated that older adults anticipate hardships in using this technology and therefore show also lower scores in the intention to use. However, the overall acceptance scores increase after 80, especially in the perceived usefulness (Figure $2 \mathrm{~b}$ ). This is also reflected in smaller effect sizes between the age group below 20 and above 80 (Cohen's D=0.18) (SI table1). Concluding, data shows the highest acceptance in the age group below 20 years of age, with a general decrease of acceptance until 80. Despite the general trend, there is an increase in the oldest age group above 80 years, which can be interpreted as increasing mobility needs rather than trust.

The effect of condition. The next step investigates how the experimental condition influences TAM factors in the combination mentioned above of MANOVA and LDA. The central hypothesis for the condition is that a traditional taxi with a human-like avatar elicits the highest scores in the TAM, in contrast to expected low values in the radio conditions due to the lack of information about the traffic situation. AVAS should compensate for this by verbal feedforward and feedback and thus show higher scores across all items than the radio condition but lower than the Taxi. Overall It can be shown that the condition has a significant effect with Pillai's Trace $=0.00259, \mathrm{~F}(8,15672)=2.541$, and the $\mathrm{p}$-value $=0.009$. The following standardized discriminant function coefficients of the LDA show the effects of each condition on the TAM items separately. When comparing the AVAS and radio talk conditions with the taxi driver, the LDA coefficients result in -1.12 for the intention of use, 0.99 for perceived usefulness, -0.33 perceived ease of use, and 0.44 for trust, with overall small effect sizes in all comparisons (Cohen's $\mathrm{D}=<0.11$ ). While the effect on trust and ease of use are negligible between conditions, differences can be found in the intention of use and the perceived usefulness. Although the AVAS condition has a slightly higher median score in the perceived usefulness of 71 compared to 69 in the taxi driver (Figure 2 c), it decreases in the intention. Here, the AVAS condition has a lower median score of 65 than the Taxi driver with a median of 68 (Figure 2 a). It can be concluded that there are no adverse effects of the condition on the ease of use like in gender and a) TAM scores based on gender

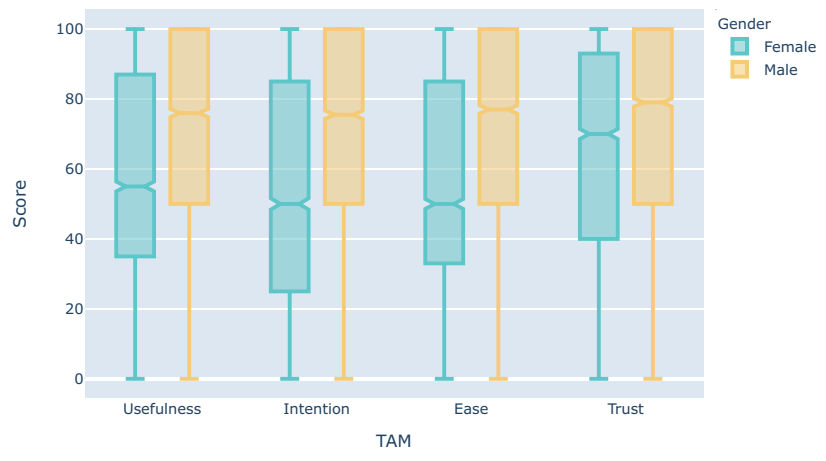

b) TAM scores based on age group
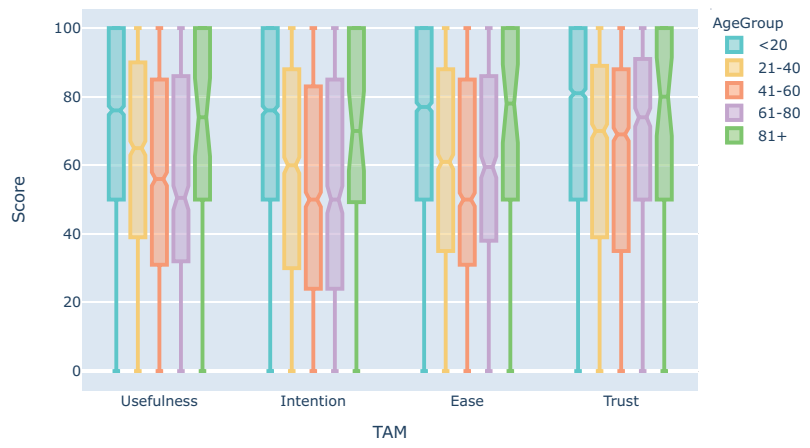

c) TAM scores based on condition

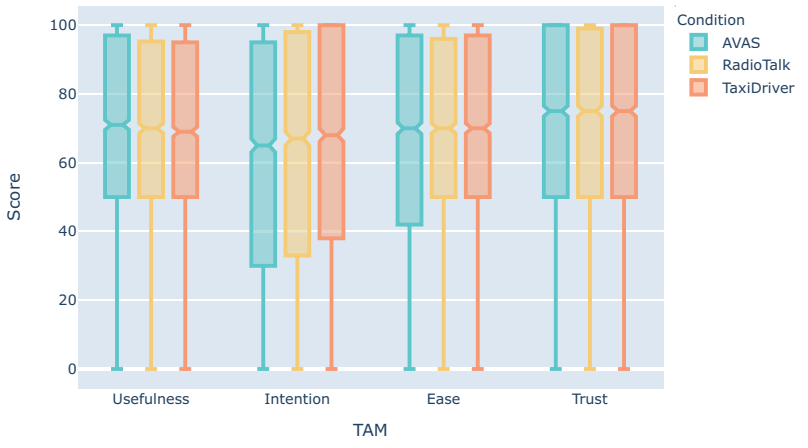

Fig. 2. Descriptive categorical plot of the mean questionnaire answers for each the main factors a) gender, b) condition and c) age Group and .

age. Still, there is a negative effect on the intention to use such technology, independently of gender and age. These results already accommodate that additional factors besides age and gender negatively influence the intention of use. This observed effect could also be due to technology aversion, which has already been mentioned in the effects of age and gender. It can be summarized that there is a small positive effect of in-car communication methods on accepting self-driving cars regarding the ease of use and a small negative effect regarding the intention of use.

The Interaction effect of gender and age group. While investigating the effects of gender and age group, and condition, it becomes clear that these factors do not explain all variance observed in the data. A significant interaction ef- 
TAM scores based on gender and age interaction

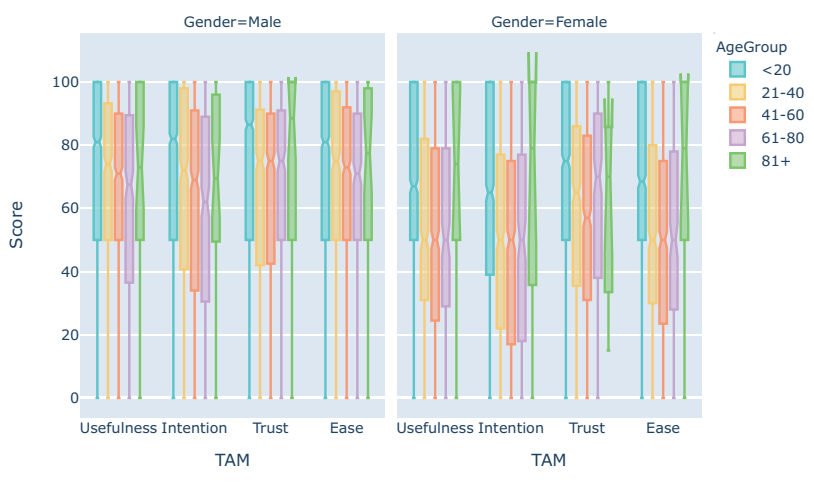

Fig. 3. Mean of answers for questions regarding usefulness, intention, trust and ease for age group and gender combined

fect of gender and age group with Pillai's Trace $=0.00498$, $\mathrm{F}(16,31352)=2.441$, and the $\mathrm{p}$-value $=0.001$ was found. According to the discriminant function coefficients of the LDA, there is a negative effect for the intention of use and perceived ease of use (both -0.73) and a positive effect on the perceived usefulness (0.22) and trust (0.55). Here, the effect sizes are most notably between the age groups 20 to 60 years compared to 20 years and above 60 for each gender (SI Table 1). These results support the findings of the previous analyses on gender and age. In addition, it could be shown that the interaction of gender and age has a significant influence on the acceptance of self-driving vehicles. In addition, the strongest effect sizes $(0.5<$ Cohens' $\mathrm{D}<=0.9$ ) are from comparing female participants of the age between 20 to 60 against the male participants in the same range. Participants of the ages below 20 have the highest TAM scores, and females between the ages of 20 to 60 years show the lowest TAM score and acceptance. Although there is a decrease in all TAM factors in both genders for ages between 20 to 60 years old compared to the below 20 years, female participants show stronger decreases in TAM scores (Figure 3 ). This accounts especially for the intention of use and perceived ease of use. Once again, as age increases for people between ages 20 to 80 , we can also observe TAM scores. In conclusion, gender and age group interaction significantly affect all TAM factors, specifically negative influences on the intention of use and perceived ease of use of the self-driving car, but a positive effect on perceived usefulness and trust. This means that although the self-explaining car is seen as useful and trustworthy, there are still other hidden factors that decrease the ease of use and the intention to use. Consequently, the demographic factors have much more impact on acceptance, i.e., the positive influence on perceived usefulness and trust scores in a self-explanatory vehicle is by far not sufficient to compensate for the negative influence of demographics on ease of use and, accordingly, intention to use.

\section{Discussion}

With questionnaire data from a considerable sample of participants, we gained insights into the influencing factors of acceptance of self-driving cars. We investigated participants' attitudes towards three types of in-car communication: a selfdriving car with a feed-forward anthropomorphic explanatory system, a self-driving car without any explanatory system, and a traditional taxi driver. In the multivariate variance and its follow-up LDAs, we observe a clear difference between men's and women's scores over all items. While male participants are more positive and accepting towards self-driving cars, females consistently rate lower over all ages, specifically regarding the perceived ease of use. Regardless of their gender, participants show decreased acceptance in the midage group between 40 and 60 . Overall it is shown that the highest acceptance scores are among people younger than 20 years and above 80 years, with an apparent decrease of acceptance scores for the age groups in between. Although middleaged participants believe that self-driving cars are trustworthy and useful in general, they do not perceive this technology as easy to use or intend to use self-driving cars. It is fair to assume that this difference in perceived ease stems from the attitude towards the technology since participants only observe the experimental trial as passive passengers in the car with no means to intervene in the car's actions, independently from the shown condition. Furthermore, the feed-forward in-car communication method does not reduce the perceived complexity of operating self-driving cars, nor does it improve the intention to use them. Still, a self-explaining feature helps build trust and perceived usefulness, while other factors instead modulate perceived ease of use and intention of use. These reservations possibly arise from computer anxiety of technology self-efficacy (28-30), which are not investigated in the presented study.

Prior research could show that improved communication between car and passenger-only increases trust when the car states why it performs a particular action, rather than stating its doing (20). Additionally, we can state that such feedforward communications do increase trust but will not increase the general user's intention of using the car. Here, it can be stated that implicit and prior perception from the participants' side modulates the perceived ease. This effect might be due to the additional cognitive load that this method of communication would burden the user with (31). On the other hand, the anticipation might be biased with a negative experience of prior interactions with the computer system (32), which would explain the low scores in the ease of use and intention to use and the higher scores for the oldest participants. In this case, the increase in acceptance among participants older than 80 is not assumed to be due to more trust towards new technology in general, or a higher computer self-efficacy, but rather due to the increased need for mobility which is highly influential on their well being rather than trust on the technology alone as shown in previous research $(33,34)$. Still, more investigation is needed to determine the root of the observed influence of the in-car communication method on the intention of use when designing 
the communication system for a specific goal gender and age group.

Due to the nature of the experiment within a public exhibition and a significant number of visitors, the technology acceptance questionnaire used in this study was a simplified version and might not grasp the entire aspect and spectrum of factors that modulate acceptance, such as technology self-efficacy, which might play an essential role in perceived ease of use. Furthermore, the questionnaire was also translated into the German language, and we could not validate the questionnaire before use in the experiment. Although part of the variance in the data might be due to the translation, such effect is thought to be minuscule and negligible since our main findings align with that of the previous works $(8,17,34,35)$. Another point of criticism could be that the experimental time was limited to 90 seconds, and each participant observed only one of the experimental conditions. However, this experience is already able to investigate participants' acceptance toward other in-car communications in self-driving cars. Additionally, the vast amount of data gathered by the experiment allowed for an entirely data-driven analyses. Therefore, the results of this study are valuable for understanding the population's acceptance of self-driving cars, despite its limitations.

Despite the apparent significant difference between people of different age groups and gender, it is still possible to describe them in three distinct groups. The first group is those participants with an overall highly positive attitude toward any self-driving car. This group includes participants of the ages below 20 years, who consistently have the highest acceptance scores. This group can be summarized as the techsavvy young generation (36). Questionnaire data shows that they will use any self-driving cars, regardless of in-car communication. This attitude is thought to stem from computers, and smart devices are already an important part of their life. Furthermore, even if they are not necessarily actively into new technologies, they are grown up with and used them. The second immediately recognizable group with a relatively high acceptance score is the older adults above 80 years. Those who need self-driving cars to continue a healthy lifestyle can still successfully conduct their daily commutes since they cannot reliably drive a car alone due to old age. They are once more able to freely commute and stay independent through the use of self-driving cars. These elderly generations appreciate the in-car communication systems as it increases their trust in the self-driving car they are using. The third outstanding group is the mid-aged, mainly female skeptics. This group is defined by a lower appeal toward selfdriving cars and the lowest ratings in the perceived ease of use. Even more, if they intend to use a self-driving car, they instead prefer a self-driving car that only does the job, namely transporting them to their destination. It might be possible to improve the acceptance of this group by improving their perceived usefulness and giving them the option to choose the method of in-car communication. In the end, we can identify three major groups that can be highly influential on acceptance, thus integration of self-driving cars into our societies; the tech-savvy young people, elderly in need of this technology, and middle-aged female participants with the low acceptance that appear would rather walk than use self-driving cars in the near future.

There are extra factors that modulate the perceived ease of use of self-driving cars that are not directly investigated in the presented study. Authors believe that observed low scores in ease of use could be interpreted by computer anxiety and technology self-efficacy $(37,38)$. This particularly applies to females compared to men (39). Since modern cars are essentially computer systems that can move and transport people, the findings regarding computer anxiety and self-efficacy can directly be transferred from computers. Furthermore, the low perceived ease of use among female participants and its connection to the intention of using technology is in line with Tarhini's (40) work. He could show that female students put more value in ease of use when it comes to using elearning systems. Although computer self-efficacy may be a culturally influenced phenomenon, it is worth investigating this matter, especially regarding self-efficacy when using computer-based technologies. Since computer self-efficacy and increasing the sense of ease in operation are key factors in improving acceptance of self-driving cars explicitly among the middle-aged female population.

To answer how a self-driving car should interact with the passengers to increase acceptance, based on the findings of this study, there seems to be no generalized way of optimal communication between passengers and self-driving cars. In this study and previous work $(17,20)$ it could be shown that a feed-forward explanatory and anthropomorphic system is beneficial for the overall trust as well as perceived usefulness in self-driving cars. However, demographic factors play a more crucial role in the intention to use this technology. In other words, it is more important who the user is than what the car is communicating to its passengers. Therefore, any in-car communication method or similar assisting systems should be highly individualized. The young generation will use self-driving cars regardless of the in-car communication methods. However, it is evident that the older generation unarguably will benefit from a transparent in-car communication system such as the one introduced in this study. It will increase their perception of ease of use and trust in the self-driving car. Thus the authors believe the self-driving car's in-car communication system should mainly focus on the younger and elderly generation who benefit from this and allows a certain degree of customization (such as the option to turn off this feature) for middle-aged customers.

Indeed, the current study could not investigate all aspects of the acceptance regarding self-driving cars. Short experiment time and simplified questionnaires leave room for many possible further investigations. It is already evident that perceived ease of use is a significant factor in the overall acceptance of self-driving cars and demographic factors highly modulate it. Therefore, it is worthwhile to understand what elements cause these differences in perception of ease toward an identical piece of technology among different demographics. Furthermore, more prolonged exposure to self-driving 
cars combined with the complete TAM2 questionnaire might give better insight into acceptance and trust regarding selfdriving cars. Additionally, the author's conclusion, namely the role of individualization and customization of in-car communication to improve acceptance, should be further investigated. There is little to no doubt self-driving cars are a part of future transportation. Their environmental and social benefits are already great incentives to try and integrate this technology into our societies. However, they will never fully be integrated until a significant part of society will accept and intend to use this technology. Therefore, there will be a mix between traditional car driving and self-driving cars until it fully transits towards the latter. It is the role of manufacturers and researchers to use this time to develop implementations that improve the trust and acceptance of society toward self-driving cars. Since this study has investigated the fundamental factors in acceptance, meaning the demographic factors, the results of this study can be a starting point for further research toward specific goal groups. This will enable a customer-directed design that meets adversarial needs and facilitates the integration of self-driving cars.

\section{Materials and Methods}

To be able to make statements about the general attitude of an entire population, a large number of subjects in a wide age spectrum is required. In the study at hand, we present a dataset of 8599 participants (3407 female) answering an adapted technology acceptance questionnaire (TAM V2). Remaining participants identify as intersex who have been excluded from the analysis due to the imbalance in the number of participants in this category compared to those identified as either male or female (Figure 4). We use a setup inside the MS-Wissenschaft, a traveling exhibition ship of German's ministry of research and education, across 28 cities in Germany and three cities in Austria for six months.

During the drive, participants' head movements were recorded, and they were asked to answer an adapted questionnaire of the technology acceptance model (TAM) after the trial (30). We gathered behavioral data of 21,356 participants of all age groups during the six months of data acquisition. Around 8,774 answered a simplified and adapted questionnaire of the technology acceptance model. Only the questionnaire data will be covered in this manuscript. Using non-parametric statistics, we see clustering in ages showing an effect of age on acceptance of self-driving cars and a generation gap in the trust towards this new technology.

Experimental setup. The used setup consists of two HTC Vive pro-VR HMDs without controllers and lighthouses version 1 for tracking head position and rotation while seated in the experimental setup. The VR computers were equipped with Nvidia Geforce RTX 2080Ti GPUs, 16Gb of RAM, and Intel Xeon W-2133 CPU @ 3.60Ghz core clock. Additionally, the setup uses two raspberry pies and touch monitors for web-based questionnaires. For analyses, Python 3.6, pandas 0.24 .2 , NumPy 1.16.4, statsmodels 0.10.0, as well as SPSS 29 were used. All plots were created using Matplotlib 3.1.0
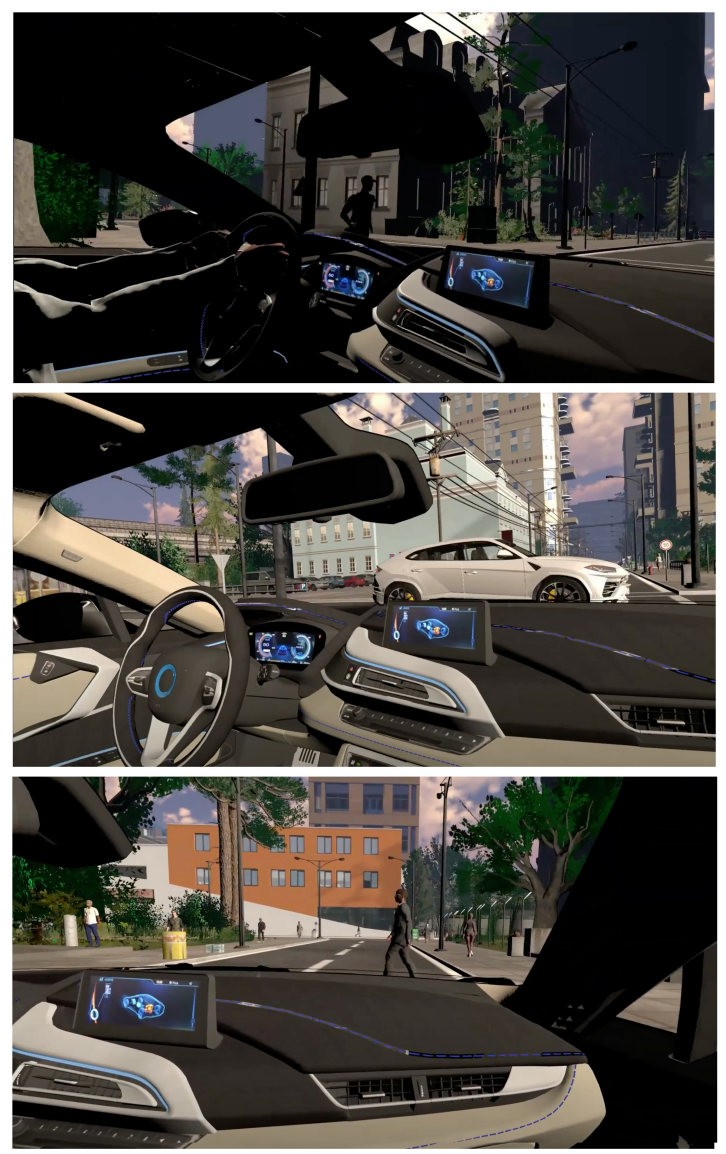

Fig. 4. Scripted critical events during the ride from top to bottom: Pedestrians running on the street from left to right, fast cars cutting in the self-driving car path, and pedestrians walking in the middle of the road.

combined with seaborn 0.9.0. Data-driven preprocessing was performed with the OPTBIN algorithm (Knuth, 2013) using histogram-based age binning. None of the participants' answers were removed from the dataset.

Experimental design and conditions. At the beginning of the experiment, participants were informed about the purpose of the study and the data gathered during the experiment, both in visual and auditory form. Here, participants had time to take off the headset. By choosing to continue with the experiment until the end, they provided us with their informed consent. Additionally, the participants could stop the experiment by taking off the VR HMD, and only the participants who finished the experiment were presented with the final technology acceptance questionnaire.

The modified version included three questions from original TAM 2 in perceived usefulness, perceived ease of use, the intention of use, and one additional question on perceived trust. It also included questions on age, gender, aviophobia, driving experience, amount of gaming hours per week, and the number of exposures to virtual reality before the experiment.

Analysis of the data. The questionnaire data ( 1 condition in 1 trial per test subject) is analyzed in the present study. To better understand and investigate the extent to which each of 
the factors of gender, age group, and driving condition affect each of the four aspects of the intention of use, perceived usefulness, ease of use, and trust, a one-way multivariate analysis of variance (MANOVA) has been performed. MANOVA tests the optimal linear combination of dependent variables to find significant effects. In addition, to investigate if differences in the observed driving condition, gender, or age group would lead to a change in attitudes toward self-driving car using the four TAM aspects, a one-way MANOVA has been performed for the four TAM aspects modeled with respects to the observed driving condition, gender, and age.

While conducting the MANOVA on the mentioned model, results are interpreted using Pillai's Trace test statistic. Like the other available test statistics, namely Wilk's lambda, LawleyHotelling's trace, or Roy's greatest root, Pillai's Trace uses estimated F-Values to test significance $(=0.05)$. However, unlike others, it is known as a robust test statistic toward nonnormalities. Therefore Pilli's Trace adds an extra layer of protection against false positives (Finch and French 2013), in other words, falsely assuming a significant effect where there is, in fact, none. Hence the conducted MANOVA indicates a significant effect for all mentioned factors of observed driving condition, gender, and age group. Knowing that they are significant, although valuable, is not enough as it is more important to understand how different categories within each factor, E.g., male or female in gender, affect the four TAM aspects compared to one another. This can be done by conducting a separate one-way analysis of the variance (ANOVA) for each of the factors and looking into the different effect sizes (Cohen's D and Hedge's G) using estimated means and standard deviations reported for each category within that factor. Nevertheless, ANOVA, unlike the previous method, can only be done on a single independent variable. Therefore an extra step is essential to summarize the four TAM aspects for each participant into a single value representing all these aspects.

The best way to combine the four TAM aspects into one value is by multiplying each aspect's score for a given participant by a corresponding weight and adding them all together to get a single value. To do so performing a linear discriminant function for each factor. The discriminant function, in return, will yield a different raw coefficient for each of the TAM aspects concerning the given factor. The linear discriminant analysis (LDA) and detailed interpretation of these extracted coefficients are described in detail in the following section. Here these raw discriminant function coefficients can be used as weights to drive the single value representation of the four TAM aspects for the given factor on a per-participant basis.

Three one-way ANOVA is then performed for gender, age group, and driving condition to calculate the effect sizes. Finally, the resulting estimated means and standard deviations for each level within the factors are used to calculate Cohen's D and Hedge's G. Although both of these effect sizes are nearly the same and interpreted in the same manner based on Cohen's suggestions (quote), in contrast, Hege's G also considers the sample sizes of the groups under comparison while calculating the effect sizes. Therefore both effect sizes have been used to interpret the results.

\section{Acknowledgment}

The authors would like to thank all involved members of the WestDrive team that helped building software and hardware as well as acquiring data. Namely, we would like to thank: Johannes Max Pingel and Sumin Kim for helping to organize and commenting the experimental code, again Johannes Max Pingel for maintaining hardware during the duration of the exhibition. Deniz Gün and Thomas Klein for implementing the supplementary material into the exhibitions, as well as Philipp Spaniol for designing and building the exhibitions and lastly Marc Vidal de Palol and Hristofor Lukanov for implementing the Questionnaire, supplementary material, and server structure. Without the support of all these wonderful members of the institute, this project would not have been realized.

\section{Authors Contribution}

FNN and MW developing and designing and conducting the experiment as well as analysis of the data. $\mathrm{AK}$ and $\mathrm{AC}$ assisted in the analysis of the data as well as reviewing the manuscript, KL and MV has developed the questionnaire used in the experiment. PK and GP supervised the project and reviewed the manuscript

\section{Conflict of Interests}

The authors declare that the research was conducted in the absence of any commercial or financial relationships that could be construed as a potential conflict of interest.

\section{Funding}

This work is funded by the University of Osnabrück in cooperation with the graduate college "Vertrauen und Akzeptanz in erweiterten und virtuellen Arbeitswelten" (FN), the German Federal Ministry of Education and Research for the project ErgoVR (Entwicklung eines Ergonomie-AnalyseTools in der virtuellen Realität zur Planung von Arbeitsplätzen in der industriellen Fertigung)-16SV8052(AK), as well as from the GMH foundation (MW).

\section{Datasets}

Code of the entire experiment conducted in this article is available in project-westdrive Gitlab repository under creative common license. furthermore all analysis scripts and output resuts, as well as raw data and demo video are available at OSF under creative common license.

\section{Bibliography}

1. Yanja Dajsuren and Mark van den Brand. Automotive software engineering: Past, present, and future. In Yanja Dajsuren and Mark van den Brand, editors, Automotive Systems and Software Engineering: State of the Art and Future Trends, pages 3-8. Springer International Publishing, Cham, 2019.

2. Ahmed Ziad Benleulmi and Thorsten Blecker. Investigating the factors influencing the acceptance of fully autonomous cars. In Digitalization in Supply Chain Management and Logistics: Smart and Digital Solutions for an Industry 4.0 Environment. Proceedings of the Hamburg International Conference of Logistics (HICL), Vol. 23, pages 99-115, 2017.

3. Mark Ryan. The future of transportation: Ethical, legal, social and economic impacts of self-driving vehicles in the year 2025. Sci. Eng. Ethics, 26(3):1185-1208, June 2020. 
4. Kyriakidis M., Happee R., and Winter J.c.f. Public opinion on automated driving: Results of an international questionnaire among 5000 respondents. Transp. Res. Part $F$ Traffic Psychol. Behav., 32, 2015.

5. Chaiwoo Lee, Carley Ward, Martina Raue, Lisa D'Ambrosio, and Joseph F Coughlin. Age differences in acceptance of self-driving cars: A survey of perceptions and attitudes. In Human Aspects of IT for the Aged Population. Aging, Design and User Experience, pages 3-13, Cham, 2017. Springer International Publishing.

6. Chaiwoo Lee, Bobbie Seppelt, Bryan Reimer, Bruce Mehler, and Joseph F. Coughlin. Acceptance of vehicle automation: Effects of demographic traits, technology experience and media exposure. Proceedings of the Human Factors and Ergonomics Society Annual Meeting, 63(1):2066-2070, 2019. doi: 10.1177/1071181319631425.

7. M Raue Ward, C Lee, L D'Ambrosio, J F, and M. Coughlin, “Acceptance of automated driving across generations: the role of risk and benefit perception, knowledge, and trust. In $\mathrm{HCl}$ 2017, Lecture Notes in ComputerScience, Vol.10271, pages 254-266,. Springer, Cham, 2017.

8. Kareem Othman. Public acceptance and perception of autonomous vehicles: a comprehensive review. Al and Ethics, 63, February 2021.

9. Amin Rezaei and Brian Caulfield. Examining public acceptance of autonomous mobility. Travel Behaviour and Society, 21:235-246, October 2020.

10. Mark Ryan. The future of transportation: Ethical, legal, social and economic impacts of self-driving vehicles in the year 2025. Sci. Eng. Ethics, 26(3):1185-1208, June 2020.

11. J Hawthorne-Castro. Autonomous vehicles will be a new opportunity for marketers, 2018.

12. Daniel Howard and Danielle Dai. Public perceptions of self-driving cars: The case of berkeley, california. In Transportation research board 93rd annual meeting, volume 14, pages $1-16,2014$.

13. Rico Krueger, Taha $H$ Rashidi, and John M Rose. Preferences for shared autonomous vehicles. Transp. Res. Part C: Emerg. Technol., 69:343-355, August 2016.

14. Péter Szikora and Nikolett Madarász. Self-driving cars - the human side. In 2017 IEEE 14th International Scientific Conference on Informatics, pages 383-387, Nov 2017. doi: 10.1109/INFORMATICS.2017.8327279.

15. Sara J Czaja, Joseph Sharit, Neil Charness, Arthur D Fisk, and Wendy Rogers. The center for research and education on aging and technology enhancement (CREATE): A program to enhance technology for older adults. Gerontechnology, 1(1):50-59, September 2001.

16. M König and L Neumayr. Users' resistance towards radical innovations: The case of the self-driving car. Transp. Res. Part F Traffic Psychol. Behav., 44:42-52, January 2017.

17. Jeamin Koo, Dongjun Shin, Martin Steinert, and Larry Leifer. Understanding driver responses to voice alerts of autonomous car operations. Int. J. Veh. Des., 70(4):377, 2016.

18. Yannick Forster, Frederik Naujoks, and Alexandra Neukum. Increasing anthropomorphism and trust in automated driving functions by adding speech output. In 2017 IEEE Intelligent Vehicles Symposium (IV), pages 365-372. IEEE, June 2017.

19. Na Du, Jacob Haspiel, Qiaoning Zhang, Dawn Tilbury, Anuj K Pradhan, $X$ Jessie Yang, and Lionel P Robert, Jr. Look who's talking now: Implications of AV's explanations on driver's trust, AV preference, anxiety and mental workload. Transp. Res. Part C: Emerg. Technol., 104:428-442, July 2019.

20. Jeamin Koo, Jungsuk Kwac, Wendy Ju, Martin Steinert, Larry Leifer, and Clifford Nass. Why did my car just do that? explaining semi-autonomous driving actions to improve driver understanding, trust, and performance. Int. J. Interact. Des. Manuf., 9(4):269-275, November 2015.

21. Farbod N Nezami, Maximilian A Wächter, Gordon Pipa, and Peter König. Project westdrive: Unity city with self-driving cars and pedestrians for virtual reality studies. Front. ICT, 7:1, January 2020.

22. Fred D Davis and Viswanath Venkatesh. A critical assessment of potential measurement biases in the technology acceptance model: three experiments. Int. J. Hum. Comput. Stud., 45(1):19-45, July 1996

23. K H Knuth. Optimal data-based binning for histograms, 2013.

24. Jolynn Pek, Octavia Wong, and Augustine C M Wong. How to address non-normality: A taxonomy of approaches, reviewed, and illustrated. Front. Psychol., 9:2104, November 2018.

25. Can Ateş, Özlem Kaymaz, H Emre Kale, and Mustafa Agah Tekindal. Comparison of test statistics of nonnormal and unbalanced samples for multivariate analysis of variance in terms of Type-I error rates. Comput. Math. Methods Med., 2019:2173638, July 2019.

26. R T Warne. A primer on multivariate analysis of variance (MANOVA) for behavioral scientists. Practical Assessment, Research \& Evaluation, 19, 2014.

27. Russell T. Warne, Maria Lazo, Tammy Ramos, and Nicola Ritter. Statistical methods used in gifted education journals, 2006-2010. Gifted Child Quarterly, 56(3):134-149, 2012. doi: $10.1177 / 0016986212444122$.

28. M Igbaria and $\mathrm{J}$ livari. The effects of self-efficacy on computer usage. Omega, 23(6): 587-605, December 1995.

29. Viswanath Venkatesh. Determinants of perceived ease of use: Integrating control, intrinsic motivation, and emotion into the technology acceptance model. Information Systems Research, 11(4):342-365, 2000.

30. Viswanath Venkatesh and Fred D Davis. A model of the antecedents of perceived ease of use: Development and test. Decis. sci., 27(3):451-481, September 1996.

31. A Hamish Jamson and Natasha Merat. Surrogate in-vehicle information systems and driver behaviour: Effects of visual and cognitive load in simulated rural driving. Transp. Res. Part F Traffic Psychol. Behav., 8(2):79-96, March 2005.

32. Viswanath Venkatesh and Fred D Davis. A theoretical extension of the technology acceptance model: Four longitudinal field studies. Manage. Sci., 46(2):186-204, February 2000.

33. L D'Ambrosio, J Rudnik, C Lee, T Patskanick, and J Miller. How active older adults manage their mobility needs: Transportation insights from a panel of adults ages 85+. Innov Aging, 2020;4(Suppl 1):611, December 2020.

34. K Chen and A H S Chan. A review of technology acceptance by older adults. Gerontechnology, 10(1):1-12, January 2011.

35. Viswanath Venkatesh and Michael G Morris. Why don't men ever stop to ask for directions? gender, social influence, and their role in technology acceptance and usage behavior. Miss. Q., 24(1):115-139, 2000.
36. Emmanuel Apergis. Who is tech savvy? exploring the adoption of smartphones and tablets: An empirical investigation. The Journal of High Technology Management Research, 30(2): 100351, November 2019

37. Raafat George Saadé and Dennis Kira. Mediating the impact of technology usage on perceived ease of use by anxiety. Computers \& education, 49(4):1189-1204, 2007.

38. Gary Hackbarth, Varun Grover, and $Y$ Yi Mun. Computer playfulness and anxiety: positive and negative mediators of the system experience effect on perceived ease of use. Information \& management, 40(3):221-232, 2003.

39. David Gefen and Detmar W Straub. Gender differences in the perception and use of E-Mail: An extension to the technology acceptance model. Miss. Q., 21(4):389-400, 1997.

40. Ali Tarhini, Kate Hone, and Xiaohui Liu. Measuring the moderating effect of gender and age on E-Learning acceptance in england: A structural equation modeling approach for an extended technology acceptance model. Journal of Educational Computing Research, 51 (2):163-184, September 2014. 


\section{Effect Sizes Tables}

In the following tables all effect sizes has been calculated for the significant independent variables of the MANOVA based on Cohen's D and Hedges G, in case of big differences both measures of effect sizes has been reported. 
Table 1. Effect Sizes between different age groups on Intention to use, perceived usefulness, perceived ease of use and trust. Numbers in the table present the Cohen's D and in the case of difference Hedges $G$

$\begin{array}{rrrrrr}\text { age group } & \text { below 20 } & 20-40 & 40-60 & 60-80 & \text { above } 80 \\ \text { below 20 } & 0 & 0.40 & 0.50 & 0.43 & 0.17 / 0.2 \\ 20-40 & 0.40 & 0 & 0.10 & 0.01 & 0.18 / 0.20 \\ 40-60 & 0.50 & 0.10 & 0 & 0.08 & 0.28 \\ 60-80 & 0.43 & 0.01 & 0.08 & 0 & 0.20 / 0.22 \\ \text { above } 80 & 0.17 / 0.2 & 0.18 / 0.20 & 0.28 & 0.20 / 0.22 & 0\end{array}$

Table 2. Effect Sizes between different condition on Intention to use, perceived usefulness, perceived ease of use and trust. Numbers in the table present the Cohen's D and in the case of difference Hedges $G$

$\begin{array}{rrrr}\text { condition } & \text { AVAS } & \text { RadioTalk } & \text { TaxiDriver } \\ \text { AVAS } & 0 & 0.05 & 0.11 \\ \text { RadioTalk } & 0.99 & 0 & 0.06 \\ \text { TaxiDriver } & 0.11 & 0.06 & 0\end{array}$

Table 3. Effect Sizes between different combination of gender and age group on Intention to use, perceived usefulness, perceived ease of use and trust. Numbers in the table present the Cohen's D and in the case of difference Hedges $G$

\begin{tabular}{|c|c|c|c|c|c|c|c|c|c|c|}
\hline Gender/Age Group & Male / below 20 & Male / $20-40$ & Male / 40 - 60 & Male / $60-80$ & Male / $80+$ & Female / below 20 & Female / 20 - 40 & Female / $40-60$ & Female / $60-80$ & Female / 80+ \\
\hline Male / below 20 & 0 & 0.30 & 0.36 & 0.39 & $0.28-0.32$ & 0.31 & 0.73 & 0.9 & $0.78-0.80$ & $0.29-0-33$ \\
\hline Male / 20 - 40 & 0.30 & 0 & 0.06 & 0.07 & 0 & 0.01 & 0.42 & 0.58 & 0.46 & 0 \\
\hline Male / 40 - 60 & 0.36 & 0.06 & 0 & 0.01 & 0.05 & 0.05 & 0.36 & 0.51 & 0.40 & 0.03 \\
\hline Male / $60-80$ & 0.39 & 0.07 & 0.01 & 0 & 0.06 & 0.06 & 0.35 & 0.51 & 0.40 & 0.05 \\
\hline Male / 80+ & $0.28-0.32$ & 0 & 0.05 & 0.06 & 0 & 0.04 & $0.39-0.41$ & $0.53-0.56$ & $0.42-0.44$ & 0.01 \\
\hline Female / below 20 & 0.31 & 0.01 & 0.05 & 0.06 & 0.04 & 0 & 0.42 & 0.57 & 0.46 & 0.01 \\
\hline Female / 20 - 40 & 0.73 & 0.42 & 0.36 & 0.35 & $0.39-0.41$ & 0.42 & 0 & 0.15 & 0.03 & $0.37-0.40$ \\
\hline Female / $40-60$ & 0.90 & 0.58 & 0.51 & 0.51 & $0.53-0.56$ & 0.57 & 0.15 & 0 & 0.11 & $0.51-0.55$ \\
\hline Female / $60-80$ & $0.78-0.80$ & 0.46 & 0.40 & 0.40 & $0.42-0.44$ & 0.46 & 0.03 & 0.11 & 0 & $0.41-0.44$ \\
\hline Female / 80+ & $0.29-0-33$ & 0 & 0.03 & 0.05 & 0.01 & 0.01 & $0.37-0.40$ & $0.51-0.55$ & $0.41-0.44$ & 0 \\
\hline
\end{tabular}

